\title{
Activités
}

1-1 | avril 2004

Varia

\section{Action et connaissance en clinique de l'activité}

\section{Yves Clot}

\section{(2) OpenEdition}

Journals

Édition électronique

URL : http://journals.openedition.org/activites/1145

DOI : 10.4000/activites. 1145

ISSN : 1765-2723

\section{Éditeur}

ARPACT - Association Recherches et Pratiques sur les ACTivités

\section{Référence électronique}

Yves Clot, « Action et connaissance en clinique de l'activité », Activités [En ligne], 1-1 | avril 2004, mis en ligne le 02 avril 2004, consulté le 01 mai 2019. URL : http://journals.openedition.org/activites/1145 ; DOI : 10.4000/activites. 1145

\section{(c) (i) (3)}

Activités est mis à disposition selon les termes de la licence Creative Commons Attribution - Pas d'Utilisation Commerciale - Pas de Modification 4.0 International. 


\title{
Action et connaissance en clinique de l'activité
}

\author{
Yves Clot \\ Chaire de psychologie du travail, \\ 41 rue Gay-Lussac, 75005 Paris. \\ clot@cnam.fr
}

\begin{abstract}
RÉSUMÉ
Dans cet article on s'interroge sur les rapports entre l'intervention en milieu de travail et la production de connaissances en psychologie. Après l'analyse de trois exemples très différents on propose un modèle pour comprendre l'architecture de l'activité.
\end{abstract}

\begin{abstract}
In this paper we discuss the relationships between transformation action in the work field and knowledgeEacquisition in psychology. We analyse three very different examples and suggest a model to understand the architecture of activity.
\end{abstract}

MOTS-CLÉ

Activité, intervention, psychologie, connaissance.

KEYWORDS

Activity, transformation action, psychology, knowledge.

Beaucoup de discussions en ergonomie ou en psychologie du travail tournent autour des rapports entre recherche et intervention. Dans cet article on restreindra le problème aux méthodes. En psychologie du travail est-il envisageable de regarder une méthode d'action également comme une méthode de connaissance? Et, parce qu'il est impossible d'aborder ici un ensemble trop vaste de méthodes d'intervention qu'il est pourtant toujours très utile de comparer (Leplat, 2000), on restreindra encore la réflexion. On se focalisera de manière intéressée sur l'une d'entre elles : peut-on envisager la clinique de l'activité simultanément comme un instrument d'action et de connaissance ? Et surtout, si oui, à quelles conditions et avec quels effets pour la définition de l'action et pour celle de la connaissance?

Pour apporter des éléments de réponse à ces questions, on propose ci-dessous au lecteur d'accepter de faire un détour. L'article qui suit est volontairement singulier. Avant d'en venir à l'exposé d'un exemple en milieu de travail ordinaire, on voudrait prendre le risque de dépayser ce lecteur par quelques comparaisons. On commencera donc par la description d'une situation singulière faite en dehors du cadre habituel de l'analyse du travail. Il s'agit d'une séquence de travail tirée de l'analyse d'une activité de mise en scène lors d'une répétition théâtrale. On poursuivra par le court exposé 
d'une expérience clinique conduite par Vygotski et Luria. On terminera en tirant quelques leçons théoriques d'une intervention à la Poste dans une perspective de psychologie de l'activité.

\section{1.- La vie comme excédent}

Olivier Besson a rendu compte avec précision du travail de répétition d'une scène de La mère coupable de Beaumarchais créé le 17 février 1990 à la Comédie Française sous la direction de J. P. Vincent. Lors d'une séance de ces répétitions de la première scène de l'Acte III, Suzanne et sa maîtresse, la Comtesse Almaviva, sont, comme le prévoit le texte, dans une attente silencieuse. Suzanne espère la révélation d'un secret. Sans pouvoir entrer ici dans les détails de l'intrigue, on retiendra l'analyse qu'O. Besson propose de l'activité du metteur en scène et des comédiennes. Le premier, J. P. Vincent, demande aux deux comédiennes d'étirer le silence un long moment pour instaurer un sentiment de malaise. La Comtesse devait apparaître pensive, la servante (Catherine Samie) l'interrogeant du regard, l'esprit actif.

Ce scénario de début d'acte, longtemps affiné était pertinent. Pourtant le travail en restait à un stade embarrassant : « les actrices, écrit $\mathrm{O}$. Besson, marquaient les intentions de jeu de façon juste, mais lourde » (1991, p. 190). Le texte leur était familier, « cependant, le bâti restait très visible ». Catherine Samie ${ }^{1}$ (Suzanne), trop fébrile face à la Comtesse, manifestait «trop de gestes, de signes d'impatience dans le corps et la voix lorsqu'elle prenait enfin la parole. Elle parlait trop vite et trop fort ». Son désir de persuader la Comtesse était « plus militant qu'insidieux ». De son côté, elle avait bien conscience que « des schémas de jeu préétablis la tenaient à distance de son texte, et qu'elle n'avait pas encore inventé la simplicité de l'impatience de ce moment là ». Le jeu des comédiennes est, à ce stade là du travail, captif de la répétition d'une lourdeur.

L'activité du metteur en scène intervient ici dans l'action en quelque sorte engourdie des actrices. J.-P. Vincent, imaginant qu'il est encore tôt le matin, " demanda alors à C. Samie d'entrer en scène avec un chandelier allumé à la main ». Atmosphère de début du jour. Mais, sans que personne n'ait pu bien sûr le prévoir, " après quelques minutes de répétition, les bougies du chandelier coulèrent, l'actrice se brûla les mains; le chandelier proposé par les accessoiristes était trop lourd pour elle. L'enjeu de la scène à cette répétition consista alors pour C. Samie à s'occuper de cet encombrant et douloureux objet. Elle en oublia d'interpréter le texte, de jouer les repères du jeu établis. Mais le travail préalable en avait fait des automatismes, au même titre que la remémoration du texte. Tout ce qui avait été construit jusque là vint dans la scène sans qu'elle y prenne garde. Détournement d'attention» (ibid, p. 190). À l'insu de l'actrice, le texte parlait à travers elle tandis que «toutes défenses levées ", écrit O. Besson, C. Samie s'appliquait à tenir son chandelier sans se brûler les mains. Le chandelier était finalement devenu l'instrument de la rencontre entre la comédienne et le texte.

Il fallut évidemment s'occuper, avec les accessoiristes, d'améliorer cet artefact incommode qu'était le chandelier. " A chaque répétition de cette scène, la question de la conception du chandelier devint désormais centrale ». En fait, pendant le dialogue, Suzanne l'éteignait entre ses doigts. Mais il brûlait depuis deux actes. Changer de chandelier pour substituer à celui qui lui brûlait les mains un autre chandelier doté de bougies neuves, froid et sans cire, s'imposa entre les actes. O. Besson note : «Une émotion juste se dégageait enfin de la scène. Elle devenait simple. Suzanne jouait un enjeu humain immédiatement perceptible : changer des chandeliers sans se brûler les mains ». À quelques jours de la première, c'était devenue la scène du chandelier : «Et plus Catherine Samie s'appliquait à ses manipulations, plus elle leur accordait de soins, plus elle entrait en contact avec son texte et avec le travail qui l'avait menée là. Ce n'était plus du travail mais de la vie. Les éléments divers qui

1. C. Samie est l'une des comédiennes les plus anciennes de la Comédie Française. 
avaient concouru à son éclosion y étaient assimilés, définitivement indiscernables. Tous y tenaient, mais dans une forme nouvelle qui les excédait. Cet excédent était ce qui donnait la vie » (ibid, p. 190).

Le détour demandé au lecteur n'est pas terminé. Mais retenons ici qu'on est bien face à une catachrèse ou, plus précisément, à une «genèse instrumentale » (Béguin, 1994 ; Rabardel, 1995). Notons cependant qu'elle prend sa source dans l'histoire collective d'une activité contrariée (Clot, 2002 a). La genèse sert de ressource dans une histoire (Clot, 2003 a). Elle développe l'activité des comédiennes en les sortant des tâches factices qui les retenaient prisonnières. Cet « allègement » de l'action s'opère par un « détournement d'attention » qui réalise la tâche initiale par l'entremise d'une activité imprévue. On a déjà eu l'occasion de montrer l'intérêt de ces "passages » d'une activité dans l'autre, de ces phénomènes de déliaison-reliaison dans d'autres contextes (Clot, 2003 a ; 2003 b).

Mais dans cet article, on poursuit d'abord un premier objectif pratique : avec cet exemple et celui qui va suivre on insiste sur le fait que l'action de l'intervenant (ici le metteur en scène) est, au bout du compte, seulement la ressource de l'activité propre des comédiennes. Il agit par artefact interposé à l'intérieur des conflits de leur activité en les déplaçant. Il prend sa part dans une transformation du cercle vicieux de la répétition d'une impasse en cercle vertueux d'une « répétition sans répétition » de l'activité (Bernstein, 1996). On reviendra, à la fin de cet article - c'est son objectif proprement théorique - sur cette dernière formulation qui pourrait bien être la définition même du développement.

\section{2.- Enjamber pour marcher}

Mais on voudrait maintenant achever le détour proposé avec un deuxième exemple encore éloigné de ce que nous désignons habituellement comme analyse de l'activité. Cet exemple a ici le même rôle que le premier. Mais en mettant l'accent sur une activité clinique de réhabilitation de fonctions vitales pour le sujet, il nous servira de transition vers l'analyse de l'action en milieu de travail que nous avons choisie. Elle aussi concerne la restauration d'une fonction vitale : la fonction psychologique du collectif dans l'activité individuelle. Mais revenons d'abord à notre deuxième exemple d'action.

Il devrait permettre de comprendre encore mieux ce qui relie de l'intérieur, en clinique de l'activité, l'observation, les hypothèses et l'action. A. Luria, l'un des fondateurs de la neuropsychologie moderne, a rapporté ainsi l'expérience de sa collaboration avec Vygotski dans son travail avec des malades atteints de la maladie de Parkinson. Les remarques de Luria commencent par le récit d'une observation surprenante : «Fait paradoxal, écrit-il, des malades qui n'étaient pas capables de faire deux pas de suite sur une surface plane pouvaient par contre monter un escalier. Nous supposions que lorsqu'un individu monte un escalier, chaque pas représente pour lui une tâche motrice spéciale. L'enchaînement régulier, automatique des mouvements de la marche sur une surface plane est remplacé par une chaîne d'actes moteurs isolés. Autrement dit, la structure de l'activité motrice se réorganise et des réponses conscientes à chaque signal isolé se substituent à la marche habituelle spontanée dont l'organisation est sous-corticale. Vygotski utilisa un procédé très simple pour mettre au point un modèle de laboratoire de réorganisation d'un mouvement de ce type. Il disposa sur le plancher des morceaux de papier et demanda aux malades d'arpenter la pièce en les enjambant. Il se produisit un phénomène étonnant. Le malade qui, un instant auparavant, n'était pas capable de faire de lui-même plus de deux ou trois pas, marcha longtemps à travers la pièce en enjambant les morceaux de papier, comme s'il montait un escalier. Il y avait une compensation possible des troubles moteurs à partir d'une réorganisation des processus psychiques à l'oeuvre lors de la marche. L'activité était transférée du niveau du système sous-cortical, siège de la lésion, au niveau mieux conservé du cortex » $(1985$, p. 167). 
Cette citation un peu longue n'a pas pour objet bien sûr une discussion sur la maladie de Parkinson. Depuis les années 30 on sait que la recherche a fait du chemin. Elle vise plutôt à éclairer une démarche qui cherche encore une fois à mobiliser chez le sujet des capacités insoupçonnés. Et elle s'y prend en organisant l'activité avec les sujets concernés selon deux principes complémentaires. Le premier est que l'action opère dans l'activité de l'autre par des «moyens détournés » : l'exemple précédent du chandelier vaut bien celui des «morceaux de papier». Le deuxième est celui de la «migration » du fonctionnement du sujet dans de nouveaux contextes de réalisation et d'exécution. L'activité, écrit significativement Luria a été «transférée » pour pouvoir enjamber. C'est un subtil travail de transit qui est entrepris là. Sa portée pour l'action ne peut être sous-estimée. Car la restauration fonctionnelle de la marche chez le patient — même momentanée dans ce dernier cas résulte du passage d'une activité dans une autre activité. Certes, avec ce deuxième exemple, nous nous trouvons devant un « transfert » dans l'activité du cerveau lui-même, du système sous-cortical au cortex sous l'effet d'une transformation de l'activité réelle du sujet. Mais ce processus de migration fonctionnelle n'est pas local. Il est structurel dans l'activité humaine et d'autant plus fréquent qu'on s'éloigne des supports physiologiques de cette activité en direction des activités psychiques et sociales elles-mêmes.

\section{3.- Répéter sans répéter}

En effet, les compensations possibles dans la réalisation d'une activité peuvent alors bénéficier de «systèmes fonctionnels» d'exécution de moins en moins assujettis à des organes spéciaux. Vygotski, en citant Sherrington, notait déjà qu'on peut « considérer chaque muscle, chaque organe qui travaille comme un chèque au porteur dont peut s'emparer n'importe quel groupe de récepteurs » (Vygotski, 2003, p. 75). Mais les instruments symboliques et techniques, mots et outils, sont bien sûr encore moins strictement ajustés et asservis à une fonction unique. Même si les outils sont tout autrement soumis aux propriétés physiques du monde que le langage — lequel, justement, en affranchit spécialement les sujets - il reste ce fait : la non-spécialisation technique et symbolique est une caractéristique propre de l'humain. C'est là un atout majeur pour notre action. Aussi bien l'usage du chandelier que celui des morceaux de papier, chargés d'une fonction imprévue, le montrent excellemment. Heureusement, l'homme n'adhère jamais immédiatement à son milieu. On pourrait même dire qu'en matière d'adaptation, le parfait ou le fini c'est le commencement de la fin, pour parler comme Canguilhem (1984, p. 197). C'est la possibilité d'obtenir un résultat par des procédés entièrement différents et celle de se livrer à la même occupation pour répondre à des pré-occupations changeantes ou même opposées, qui signalent finalement la santé d'un sujet.

Comme Luria a pu le montrer avec beaucoup de rigueur dans une œuvre qui reste à découvrir en France, c'est là l'esprit général d'un travail clinique instrumenté : l'établissement patient et risqué par le sujet lui-même des liaisons et re-liaisons entre ses activités ; liaisons susceptibles de le faire accéder, en s'appuyant sur le clinicien, à ce que ses troubles ont de plus concret et qui pourtant souvent lui échappe. Avec le secret espoir que ce sujet puisse tirer, des obligations que l'activité du clinicien lui fixe au travers des dispositifs techniques qu'il manie, des ressources qu'il ne soupçonnait même pas. Ainsi, souvent par des «moyens détournés », le cercle des liaisons morbides qui incarcère son action peut se ré-ouvrir. Et ce pour éventuellement parvenir à transformer son vécu en moyen de vivre une autre vie. Ce sont là les enseignements qu'on peut tirer du travail clinique de Luria dans son ensemble (1995).

Ces enseignements pour l'action n'ont rien d'analogiques. S'ils ont pu être validés en analyse du travail c'est au nom de raisons théoriques précises : il n'y a pas de fixation intangible d'un fonctionnement à son tissu contextuel, autrement dit, pas d'attachement définitif entre une activité, une action, et l'opération qui les réalise. Léontiev a bien montré l'importance de ce fait (Leontiev, 1984). On peut donc « répéter sans les répéter » les fonctionnements psychologiques. L'intervention de l'ana- 
lyste du travail peut alors en faire l'un de ses objectifs d'action privilégiés. Ce jeu ouvert de l'attachement $\mathrm{du}$ sujet à ses propres réalisations et de son détachement possible au travers d'autres réalisations - jeu que la vie ordinaire comme la pathologie se charge trop souvent de refermer est peut être un ressort central de l'action en clinique de l'activité mais pas seulement (Béguin \& Pastré, 2002).

\section{4.- Revoir l'activité}

Il nous faut donc maintenant en venir à l'exposé de notre exemple en situation de travail. Dans une intervention auprès des facteurs de La Poste, et dans un cadre méthodologique précis qu'il n'est pas possible de décrire ici (Clot \& Faïta 2000 ; Clot, Faïta, Fernandez, \& Scheller 2001), nous avons pratiqué des auto-confrontations croisées. Il s'agit de demander à des professionnels différents de commenter, également en présence de l'intervenant, des images vidéo de l'activité d'un collègue avant que celui-ci ne commente les siennes. Ces auto-confrontations supportent leur engagement réciproque dans des dialogues professionnels sur des gestes du travail ordinaire. On peut les définir comme des artefacts méthodologiques que nous cherchons à transformer avec eux en instruments effectifs de développement pour leur activité. Elles sont donc des moyens d'agir dans leur activité.

L'appropriation par les sujets de ce genre d'exercice organise une sorte de plurilinguisme professionnel orchestré contre toute canonisation de l'activité ordinaire afin de provoquer sa modulation dans l'échange. Du coup cette clinique de l'activité ne s'arrête pas sur l'activité observable pour et par la seule analyse. Bien qu'elle retienne toutes les leçons de l'ergonomie en matière d'observation du travail concret (Clot, 2002 a ; Leplat, 2000 ; Rabardel, 1995), son objet est davantage l'activité « au carré », celle qu'on observe quand les intéressés dirigent leurs analyses sur leur propre activité examinée ensemble. C'est le contenu de l'activité d'analyse dirigée vers l'activité arrêtée sur l'image qui est l'objet de ce genre de psychologie du travail. Par l'entremise du commentaire sur ce qui a déjà été fait on contraint les sujets à se référer à l'activité filmée non pas comme à une chose faite mais pour agir avec elle et sur elle afin de la « refaire ». En lui donnant, là aussi, une forme nouvelle qui excède ce qui a déjà été fait, sachant que c'est cet excédent qui donne la vie comme on l'a vu dans le premier exemple ci-dessus. Et ce dans la perspective d' «enjamber» les auto-confrontations croisées en direction de l'activité réelle.

On regardera donc ici les images filmées sous un certain angle : c'est là un outil de description des actions conçu comme un «moyen détourné » pour remettre le travail sur le «métier ». Non pas que leur fonction d'observation de l'activité pour nous soit écartée. Mais nous cherchons auprès de nos interlocuteurs à provoquer le développement de cette fonction chez eux. Et ce, en organisant sa migration, son transfert : notre objectif est de changer les protagonistes de l'observation et de placer les travailleurs concernés en position de devenir ensemble des observateurs de leur propre activité. Mieux, en position de re-devenir, au moyen de controverses professionnelles réglées, les « auteurs » d'une activité « revue et corrigée » ensemble. Ce faisant, on le mesurera plus précisément grâce à l'exemple qui suit, c'est la restauration d'une fonction qui nous occupe : la fonction psychologique du collectif dans l'activité personnelle.

\section{5.- L'exemple des facteurs : du tri à la tournée}

Il arrive que les dialogues réglés dont on vient de dire l'esprit confrontent un professionnel très expérimenté et un apprenti ou un quasi-apprenti. C'est le cas particulier de l'exemple qui suit, entre des facteurs de la Poste. L'auto-confrontation croisée que nous allons utiliser réunit un « rouleur », c'est-à-dire un non titulaire qui remplace un titulaire sur sa tournée, et un facteur ancien dans le métier. Dans cette situation très proche d'une situation d'apprentissage professionnel, l'expert qui a 
alors 25 ans d'ancienneté est confronté à un jeune «rouleur» débutant (Clot, Scheller, Caroly, Millanvoye, \& Volkoff, 2000). Les images le présentent sur une position de coupage / piquage où sont mobilisées les techniques du tri devant des casiers de lettres. Le geste du débutant, qui ne connaît pas l'organisation des casiers mise au point par le titulaire absent en fonction de ses habitudes en distribution sur la quartier, est une pénible hésitation. La lettre à la main, il parcourt toutes les cases un peu comme s'il battait la mesure, privé de tout repère. Consterné de voir ainsi son collègue perdre un temps précieux qu'il lui faudra payer d'un allongement démesuré de sa journée de travail au retour de la distribution dans le quartier, mesurant ainsi la solitude désespérante de celui-ci et l'abandon dans lequel il est, qu'il côtoie pourtant chaque jour sans la voir, il est d'abord tenté par une attitude défensive. Il explique alors à son jeune collègue que l'apprentissage a toujours été difficile pour tous, que c'est une épreuve inévitable à la quelle on doit se mesurer pour en sortir professionnellement grandi.

Mais le dialogue, comme on le verra plus bas, réserve un autre destin à ce diagnostic trop « autorisé ». Car le « rouleur » explique alors qu'il n'a pas le loisir de se consacrer à cette épreuve puisqu'il «tourne» en plus régulièrement entre les tournées de remplacement sans pouvoir tirer bénéfice d'automatismes chaque fois à reconstruire. Cette organisation du travail ne permet pas le type d'apprentissage dont le plus ancien a le souvenir. La stabilité n'est pas suffisante. Sous l'effet de cette résistance du réel dans le dialogue, le facteur expérimenté titulaire de sa tournée se trouve poussé à repenser le problème. Devant cet obstacle dont il prend alors l'exacte mesure il se souvient d'un geste autrefois réalisé par lui dans le bureau, geste dont il rapporte au passage la surprenante invention : lors de la chute à terre d'une baguette fixée au casier, baguette affichant les étiquettes classant les quartiers, l'idée lui était venue, avec un collègue, de disposer autrement, de l'autre côté de la baguette, d'autres étiquettes établies en fonction du travail du rouleur. Ainsi la baguette à deux faces, retournée par catachrèse, pouvait afficher alternativement, au besoin, la tournée du titulaire saturée d'astuces - et celle du rouleur - libérée de l'opacité de critères encore largement étrangers à son expérience. On identifiera sans peine ici encore une genèse instrumentale enracinée dans une activité contrariée (Clot, 2003 a).

Pourtant une surprise nous attendait : ce retournement de la baguette ne s'est pas finalement imposé comme un attribut du genre professionnel (Clot \& Faïta 2000) de ce milieu. La catachrèse n'a pas fait école. Fait d'autant plus étonnant que, à l'occasion de cette confrontation dialogique, ce geste était potentiellement rapatrié dans les possibilités du métier. Il aurait pu enrichir le répertoire générique du bureau et, par un choc en retour, fournir de nouvelles ressources mobilisables pour le jeune collègue. Ce geste réveillé par l'activité contrariée d'un jeune apprenti pouvait devenir un instrument de « régénération » du métier commun. Et pourtant l'analyse du travail poursuivie a montré que cette « régénération » n'est pas passée par le canal de ce geste là. En comprenant pourquoi on comprendra peut-être mieux les paradoxes du développement.

\section{6.- De l'individuel au collectif}

Pour ce faire, sans pouvoir ici présenter les images vidéos de l'auto-confrontation croisée entre les facteurs, on retiendra seulement un segment de l'échange (qui précède la redécouverte de la baguette) sur un objet précis : un « arrêt sur image » significatif des déboires professionnels endurés par le jeune « rouleur », resté seul au " piquage » après le départ en tournée de ses collègues. Il est prisonnier du cercle vicieux de l'accumulation du retard. C'est ce « rouleur» (ci-dessous désigné par M.) qui est engagé dans l'autoconfrontation croisée avec le facteur expérimenté (désigné ci-dessous par J.). Nous reproduisons la séquence :

1. Chercheur (désignant l'image-support): Qu'est-ce que ça fait quand on laisse le rouleur derrière ? On ne peut rien faire? 
2. J. : Ben, quelque part, c'est sûr que ça fait mal au cœur, mais quelque part, il y a le pour et le contre, parce qu'enfin, moi, je le vois comme cela, maintenant, il y a certaines personnes, plus on les aide, moins elles vont réussir à s'en sortir, parce qu'on est tous passé par là, jusqu'au jour finalement où il y a une prise de conscience et on se dit ça suffit, je me calme, je m'énerve plus, je partirai peut-être la dernière, mais un jour ça va s'améliorer. C'est sûr pour nous, c'est embêtant, mais on est tous passé par là. Chez certaines personnes, plus on va les aider, moins elles vont s'en sortir. Alors, c'est sûr, au départ, les petits jeunes quand ils arrivent et tout ça, c'est comme un genre de test. Il y en a ils vont paniquer dès le départ, et tout ça. On va leur filer un coup de main. Il y en a ils arrivent jamais à s'en sortir. L. quand il est arrivé, mais L., on l'a aidé pendant des mois et des mois. Et ben plus on l'aidait plus il était perdu. C'est comme un genre de petit test, quand on panique un bon coup avant, c'est malheureux à dire, mais là on va leur donner un coup de main, leur classer une ou deux rues, mais faut pas que cela devienne systématique non plus parce qu'après, il y a certaines personnes qui y prennent vite goût, qui s'habituent vite et qui vont ... bon après, c'est un travail individuel quand même.

3. M. : Ben, oui.

4. J. : Donc après on en prend vite goût. Chez certaines personnes, il y a de l'abus, mais c'est... On est tous passés par là. Je me souviens, il y a vingt ans, je paniquais à mort, c'était affreux.

5. Chercheur (s'adressant à M.) :Vous voyez ça comme ça vous ?

6. M. (s'adressant à Mme J.) : C'est un peu juste effectivement ce que tu dis mais il y a l'autre côté, tu disais que c'était à double tranchant. J'ai entendu un côté mais...

7. J. : L'autre côté c'est que si tu en as marre d'être comme ça d'être le dernier, et puis tu paniques, parce que c'est intérieur. Tu peux pas paniquer devant tout le monde, ni rien du tout. À un moment tu penses que, ah, ben ça, je pars le dernier, mais je vais y arriver et demain cela ira mieux, et le surlendemain, je...

8. M. : Ben disons que quand t'es sur une tournée pendant un certain temps, OK, mais quand tu changes tous les deux jours, comment veux-tu faire?

9. J. : Ah, ben là. Moi, je ne changeais pas tous les deux jours non plus. C'était pas la même façon ...

10. M. : Je sais pas, depuis combien de temps, vingt deux ans ? Je sais pas s'il y a vingt deux ans, il y avait autant de courrier que ça.

11. J. : Les tournées étaient plus longues.

12. M. : Les tournées étaient peut-être plus longues... Mais je sais pas du tout comment c'était il y a vingt deux ans, mais c'est vrai qu'il y a de la quantité maintenant. Tout ce qu'il y a comme publicité, ça existait pas autant que ça.

13. J. (s'adressant simultanément à $\mathrm{M}$. et au chercheur) : Et puis, il y avait une solidarité qu'il y a pas maintenant. Maintenant, même nous, on est obligé de toute manière de baisser les bras, parce qu'à la limite, ce serait toujours nous. Il y a ceux qui rentrent à midi et ceux qui rentrent plus tard à qui nous donnons un coup de main, bon, on va lui filer un coup de main, mais bon, on a plus de boulot, et c'est vrai qu'on finit plus tard, on finit après les autres, mais c'est pas normal que ce soit toujours les mêmes qui aillent aider. Il y a quelques années, tout le monde filait un coup de main, tout le monde prenait au moins une case, ou deux cases, cela nous coûtait quoi, cinq minutes, ça l'avançait, ça nous retardait pas plus, tandis que maintenant, ça se fait plus, c'est la compétition.

J. aura dit aussi, pour finir :

« Je ne comprends pas. Dans ce bureau c'est devenu la course. Certains veulent finir le travail avant d'avoir commencé ... ».

Ce commentaire final du facteur expérimenté qui débouche sur une « incompréhension » de sa propre activité et de celle du collectif, est le début d'un développement imprévu : le réveil générique ne s'est pas produit où on pouvait l'attendre. Lors de la circulation de ces analyses dans le bureau, il est apparu que les sous-entendus génériques gardaient finalement dans l'ombre l'expérience éprouvante 
des rouleurs. Chacun d'eux, sans répondant pour agir, « errait tout seul devant l'étendue des bêtises possibles », pour reprendre l'expression de J. P. Darré (1995). C'est sans doute la découverte - cette fois « réalisée » par le collectif — que plus personne dans le bureau ne vivait la même histoire qui est le ressort de transformations substantielles de l'organisation du travail qui suivra : en particulier, le rétablissement des « doublures », c'est-à-dire le partage pendant un mois de la même tournée par un ancien et un nouveau. C'est de ce côté là que le développement générique a repris son chemin et non par la généralisation de la catachrèse des baguettes retournées à laquelle on aurait pu s'attendre. C'est ainsi que le genre professionnel s'est trouvé décongestionné ou encore "dégelé ». Notons ici que les modalités de ce développement n'ont rien de définitivement énigmatiques. Sans avoir pu les prévoir, on peut non seulement les comprendre mais peut-être même les expliquer. On va y revenir.

Il reste que nous avons là un exemple de réhabilitation - au moins partielle - des fonctions psychiques du collectif dans l'activité individuelle. Mais cette restauration fonctionnelle n'est pas spontanée. On peut penser que les méthodes en clinique de l'activité - méthodes historico-clinique parviennent à faire « dérailler » le discours générique « autorisé » et convenu en offrant à nos interlocuteurs d'autres solutions que celle d'ouvrir « le parapluie du genre ». Elles capitalisent, à ce titre, nombre de «moyens détournés » pour recycler l'énergie perdue dans certaines impasses, en développant le métier « au carré », autrement dit le « travail sur le travail » (Clot, 2002 b ; 2003 c). C'est là une tâche qui ne peut être accomplie qu'avec l'expérience de patients apprentissages méthodologiques. Car son atout principal est un cadre clinique dialogique qui ne peut « supporter » les imprévus de l'action qu'à la condition que le chercheur ne cherche pas à les éliminer.

\section{7.- De l'action à la connaissance}

Reste un problème. Afin de répondre aux questions posées en début d'article, il faut maintenant se demander si ces méthodes d'action peuvent également servir de méthodes de connaissance. Avant de définir à quel prix c'est possible, on voudrait insister sur notre premier résultat : la méthode d'intervention que nous venons d'examiner n'assigne pas l'action à résidence. L'action de l'intervenant prend certes sa source dans la demande du milieu intéressé pour lequel elle est d'abord seulement une ressource externe. Mais de ressource initialement extérieure, l'action de l'intervenant devient une source nouvelle d'engagement pour les travailleurs concernés ; jusqu'au moment où elle redeviendra une ressource pour leur action propre. Mais, cette fois, une ressource interne. Ce processus d'alternance fonctionnelle des sources et des ressources de l'action interdit de se faire d'elle une idée sédentaire. Personne n'en est le propriétaire exclusif. Mais elle ne peut se développer qu'à la condition que l'intervenant saisisse tout l'intérêt qu'il y a pour lui, à l'aide d'une instrumentation clinique, à seulement seconder le développement du pouvoir d'agir du collectif. Car cet intérêt nous introduit justement à la question des connaissances. En effet, c'est seulement en provoquant le développement qu'on peut l'étudier. Or, lorsque celui-ci se réalise - même sous des formes inattendues comme dans l'exemple ci-dessus - il peut alors devenir, par un choc en retour, la source de nouvelles connaissances scientifiques possibles.

Reprenons notre exemple sous cet angle. On peut mesurer l'intérêt scientifique qu'on peut trouver dans le dialogue entre les deux facteurs reproduit ci-dessus dès lors qu'on prend pour objet de recherche le développement de l'activité et ses empêchements. On le mesure à la «bifurcation dialogique » provoquée en cours d'échange, au moins à deux reprises par l'activité du jeune rouleur (tours de paroles 8 et 12). Sa résistance argumentative offre plusieurs contextes inattendus au fonctionnement discursif « refroidi » de son collègue expérimenté. Du coup, l'activité de pensée du facteur expérimenté change de destinataire et de direction. Au début de la séquence (tour de parole 2) elle est tournée vers le rouleur. À la fin, elle est tournée vers le collectif (tour de parole 13). Ce n'est plus l'action du rouleur mais celle du collectif qui est objet de pensée pour elle. C'est qu'ici, un conflit s'ouvre entre des fonctionnalités rivales du même fonctionnement discursif. En effet, il appar- 
tient alors simultanément à deux activités différentes puisque ce discours est à la fois une façon de dire habituelle abritée sous le "parapluie générique » mais aussi un moyen d'argumenter - avec une efficacité toute relative - dans l'autoconfrontation en cours. Cette discordance fonctionnelle pousse l'activité du facteur expérimenté, dans ce cadre là, à changer de palier en se déplaçant à « l'étage du collectif», objet de préoccupation alors réveillé. Ici le développement de l'activité se fait une nouvelle fois par migration fonctionnelle du « rouleur » dans la pensée du facteur expérimenté : il était l'objet direct de l'activité langagière du facteur en début de séquence mais, de but, il devient moyen " détourné » quand celui-ci le fait « fonctionner » pour questionner le travail d'organisation du collectif au-delà de la compétition entre les facteurs. Le statut psychologique du rouleur n'est donc pas immobile dans le cours des pensées du facteur expérimenté. Et ce genre de déplacement est sans doute un critère central pour identifier un développement potentiel (Clot \& Kostulski, 2002 ; Kostulski \& Prot, sous presse). Il est, en effet, la trace repérable d'un développement de fonctionnalités nouvelles pour les « organisateurs » stabilisés dans l'action habituelle de ce facteur expérimenté.

\section{8.- Le développement comme objet scientifique}

Une clinique de l'activité n'est donc pas seulement une méthode d'action et de transformation. Elle ne tourne pas le dos à la connaissance scientifique. C'est aussi une méthode de connaissance. Mieux, on voudrait montrer, qu'au contraire, elle peut apporter sa contribution — parmi d'autres — à un renouvellement de l'objet de connaissance en psychologie du travail.

Mais il faut seulement préciser que cela n'est possible qu'à la condition d'accepter de déplacer le plan d'observation : à la différence des modèles traditionnels d'analyse, son objet n'est pas d'abord l'inventaire des invariants de l'action. Il s'agit moins de repérer les organisateurs de l'action ou les schèmes, pour parler comme Piaget, que d'étudier les organisateurs et l'organisation de la transformation de l'activité. Autrement dit moins de repérer la structure de l'activité en tant que telle que la structure de son développement possible ou impossible. L'objet de connaissance est moins l'activité que le développement de l'activité et ses empêchements. Ce sont les mécanismes de ce développement qui sont ici au centre de l'attention. Puisque «c'est uniquement en mouvement qu'un corps montre ce qu'il est », comme le note judicieusement Vygotski (1978, p. 64), c'est seulement au travers d'une expérience de transformation que l'activité de travail peut livrer ses secrets. On ne peut donc l'atteindre que par des moyens détournés : du coup, le développement de l'activité est à la fois l'objet et la méthode de cette psychologie du travail qu'on peut sûrement rapprocher d'autres tentatives (Engeström et al., 1996) mais sûrement pas confondre avec une simple ingénierie du changement organisationnel.

Pour connaître l'activité de travail il faut la mettre en mouvement. Provoquer le développement, comme nous l'avons montré ci-dessus, est le moyen de comprendre l'architecture masquée de l'activité. C'est aussi le moyen de rendre compte des lois psychologiques de son développement, de l'organisation de sa transformation. Au rang des organisateurs de cette transformation on peut compter : la fonction de variation introduite dans l'activité par son destinataire, la migration fonctionnelle des sources et des ressources de cette activité au cours de son développement, les passages interniveaux où se produisent de nouveaux buts pour l'action, ces excédents qui font du développement, comme on le soulignait plus haut, une répétition au delà de la répétition. Au bout du compte, l'étude de ces organisateurs signale un autre niveau d'investigation que celui qui est traditionnellement retenu : au-delà des « invariants » du fonctionnement, celui des « invariants » du développement luimême (Clot, sous presse).

On peut faire une autre remarque : on retrouve ici un résultat exposé ailleurs (Clot, 2003 c) : le réel de l'activité possède un volume dont l'activité réalisée par un opérateur n'est jamais que la surface. 
L'expérimentation en clinique de l'activité vise à donner du volume à cette activité réalisée. Mais elle peut servir aussi à étudier comment cette activité prend ce volume, lorsqu'elle le prend. Ainsi, dans notre exemple, on peut « suivre à la trace » le développement à l'intérieur de l'auto-confrontation croisée : partis d'une difficulté personnelle observée à l'écran par les deux facteurs ensemble, au moyen d'images utilisées pour développer une activité dialogique interpersonnelle, un expert et un novice redécouvrent dans l'échange la fonction transpersonnelle du collectif. Il la redécouvre comme « répondant » interne pour l'activité personnelle. Cette re-découverte ne laisse pas les choses en l'état. Elle débouchera sur une réorganisation ultérieure de la tâche elle-même avec le rétablissement des doublures sur les tournées. Or la tâche est sans doute ce qu'il y a de plus impersonnel dans l'activité. Ce mouvement du personnel vers l'impersonnel en passant par l'interpersonnel et le transpersonnel est sans doute l'un des processus les plus difficiles à étudier. Mais son étude est décisive car il constitue l'architecture même de l'activité de travail. On voudrait terminer cet article sur ce point.

\section{9.- Une architecture conceptuelle pour agir}

Cette architecture est mobile et développementale. C'est que l'activité est irréductiblement personnelle, interpersonnelle, transpersonnelle et impersonnelle. Personnelle et interpersonnelle, elle l'est dans chaque situation singulière toujours exposée à l'inattendu. Sans destinataire, l'activité perd son sens. Elle est transpersonnelle puisque traversée par une histoire collective qui a franchi nombre de situations et disposé de nombreux sujets à répondre plus ou moins d'elle, d'une situation à l'autre. Ce sont là les attendus génériques de l'activité, sur-destinataire de l'effort consenti par chacun. Le travail collectif de réorganisation de la tâche en assure ou non la «maintenance ». Enfin l'activité est impersonnelle justement sous l'angle de la tâche. Cette dernière est, dans l'architecture de l'activité d'un travailleur, ce qui est nécessairement le plus décontextualisé pour lui. Mais, du coup, elle est justement ce qui oriente l'activité au-delà de chaque situation particulière. Prescription indispensable, elle peut - elle devrait toujours - se nourrir des obligations génériques que les opérateurs se donnent pour la réaliser et, par un choc en retour, elle peut aussi les entretenir. Dans cette perspective, une clinique de l'activité ne perd pas de vue que la transformation du travail passe par celle de la tâche que l'organisation du travail prescrit. Elle a comme horizon le développement du pouvoir d'agir des opérateurs dans la conception continuée des tâches avec les concepteurs.

\section{0.- Conclusion}

On peut donc conclure momentanément ainsi : pour l'analyste du travail tel qu'on le conçoit ici, l'allongement du rayon d'action des opérateurs est non seulement un objet d'intervention mais aussi un objet de connaissance. En agissant, dans le temps, sur les « passages » — parfois conflictuels — des quatre dimensions de l'activité repérées ci-dessus, les unes à travers les autres, l'analyste du travail peut aussi les étudier. Vues ainsi, intervention et recherche peuvent difficilement être cantonnées dans des métiers différents. Elles sont à l'étroit dans une division du travail qu'elles débordent d'ailleurs systématiquement. Car l'action et la connaissance sont toutes les deux, selon les moments, source ou ressource l'une pour l'autre. De ce point de vue, une clinique de l'activité ne fait jamais que retrouver, à sa manière, l'expérience ordinaire de beaucoup de chercheurs et d'intervenants en analyse du travail.

Pour autant, en proposant un cadre théorique pour décrire l'architecture de l'activité — lui-même issu des travaux de Vygotski, Leontiev et Luria - on cherche (Clot, 2002 a ; Clot, sous presse) à définir un modèle qui regarde vraiment le développement comme l'excédent qui donne vie à l'activité, pour reprendre l'expression utilisée au début de cet article. 


\section{BIBLIOGRAPHIE}

Béguin, P. (1994). Travailler avec la CAO en ingénierie industrielle: de l'individuel au collectif dans les activités avec instruments. Thèse pour le doctorat en ergonomie. CNAM. Paris.

Béguin, P., \& Pastré, P. (2002). Working, learning and designing through simulation. ECCE, Proceedings of the 11th european conference on cognitive ergonomics,. Edited by S. Bagnara, S. Pozzi, A. Rizzo \& P. Wright, p. 5-12. Catania, 8-11 Sept.

Bernstein, N. (1996). Dexterity and its development. New Jersey: Laurence Erlbaum Associates.

Besson, O. (1991). Du rôle heureux de l'accident. In G. Banu, Les Répétitions. Un siècle de mise en scène. De Stanislavski à Bob Wilson. Alternatives Théâtrales, 52-53-54.

Canguilhem, G. (1984). Le normal et le pathologique. Paris: PUF.

Clot, Y. (2002, a). La fonction psychologique du travail. Troisième édition augmentée. Paris: PUF.

Clot, Y. (2002, b). Clinique de l'activité et répétition. Clinique méditerranéennes, $n^{\circ} 66$.

Clot, Y. $(2003$, a). La catachrèse entre réel et réalisé. Contribution d'un psychologue du travail. In Y. Clot \& R. Gori, Catachrèse : éloge du détournement. Nancy: PUN.

Clot, Y. (2003, b). Vygotski : la conscience comme liaison. Présentation de L. Vygotski, Conscience, inconscient, émotion. Paris: La Dispute.

Clot, Y. (2003, c). Le collectif dans l'individu ? In G. Vallery \& R. Amalberti (Dir.). Modèles et pratiques de l'Analyse du travail. 1988-2003, 15 ans dévolution. Actes du XXXVIIIème Congrès de la SELF. Paris: SELF.

Clot, Y. (sous presse). Le travail entre fonctionnement et développement. Bulletin de psychologie.

Clot, Y., \& Faïta, D. (2000). Genres et styles en analyse du travail. Concepts et méthodes. Travailler, $n^{\circ} 6$.

Clot, Y., Faïta, D., Fernandez, G., \& Scheller, L (2001). Les entretiens en autoconfrontation croisée : une méthode en clinique de l'activité. Education Permanente, $n^{\circ} 146$.

Clot, Y., \& Kostulski, K. (2002). Le fil conducteur de la sécurité. Analyse des pratiques d'ajustement dans la conduite des trains en autoconfrontation croisée. Rapport CNAM/Dédale pour la Mission de la recherche de la SNCF. Paris.

Clot, Y., Scheller, L., Caroly, S., Millanvoye, M., \& Volkoff, S. (2000). Le travail du genre professionnel comme contribution à la genèse de l'aptitude. Rapport pour la Mission de la recherche de la Poste.

Darré, J. P. (1994). Le mouvement des normes, avec Bakhtine et quelques agriculteurs. In J. P. Darré (Ed), Pairs et experts dans l'agriculture. Toulouse: Erès.

Engeström, Y, Virkkunen, J, Helle, M, Piihlaja, J., \& Poikela, R. (1996). Change Laboratory as a tool for transforming work. Lifelong Learning in Europe, 1(2), 10-17.

Kostulski, K., \& Prot, B. (sous presse). Analyse interlocutoire de la formation d'un concept potentiel. Psychologie Française.

Leontiev, A. (1984). Activité, conscience, personnalité. Moscou: Editions du progrès.

Leplat, J. (2000). L'analyse psychologique de l'activité en ergonomie. Aperçu sur son évolution, ses modèles et ses méthodes. Toulouse: Octares.

Luria, A. (1985). Itinéraires d'un psychologue. Moscou: Editions du progrès.

Luria, A. (1995). L'homme dont le monde volait en éclats. Paris: Seuil.

Rabardel, P. (1995). Les hommes et les technologies. Paris: Armand Colin.

Vygotski, L. (1978). Mind in society: the development of higher psychological processes. Cambridge: Harvard University Press.

Vygotski, L. (2003). Conscience, inconscient, émotions. Présentation d'Y. Clot. Paris: La Dispute. 